\title{
A novel functional beverage for COVID-19 and other conditions: Hypothesis and preliminary data, increased blood flow, and wound healing
}

\author{
LeBaron $\mathrm{TW}^{1,2 *}$, McCullough $\mathrm{ML}^{3}$ and Ruppman $\mathrm{Sr} \mathrm{KH}^{4}$ \\ ${ }^{1}$ Centre of Experimental Medicine, Institute for Heart Research, Slovak Academy of Sciences, Bratislava, Slovak Republic \\ ${ }^{2}$ Molecular Hydrogen Institute, USA \\ ${ }^{3}$ Medical office, internal medicine, Dallas, Texas, USA \\ ${ }^{4} \mathrm{H} 2$ Beverages Inc., PO Box 940283, Plano, TX 75094-0283, USA
}

\begin{abstract}
COVID-19 is caused by a viral infection, and can be a lethal disease to the immunocompromised. HydroShot is a popular functional health beverage that is infused with molecular hydrogen, and contains the nitric-oxide-producing amino acid citrulline. Numerous pre-clinical and clinical studies have demonstrated that the gaseous-signaling molecules, molecular hydrogen and nitric oxide, have anti-oxidant, anti-inflammatory, and immuno-modulating benefits. Although nitric oxide can directly kill pathogens, it is critical for immune function, can inhibit viral replication, and can also induce lethal cellular injury under stressed conditions. In contrast, molecular hydrogen has a very high safety profile and helps regulate nitric oxide production, metabolism, and attenuates its harmful effects. Both of these molecules are being used separately in clinical trials for COVID-19 patients. This article briefly reviews the scientific literature on these two molecules, and then presents preliminary data on HydroShot including increased blood flow and wound healing. Based on the pre-clinical, clinical, and this preliminary data, HydroShot is recommended for clinical testing/application in addition to its frequent consumer use.
\end{abstract}

\section{Graphical Abstract}

$\mathrm{H}_{2}$ and $\mathrm{NO}$ ' work together to suppress COVID-19-induced oxidative stress and inflammation, which helps to prevent multiple organ failure and death (Figure 1).

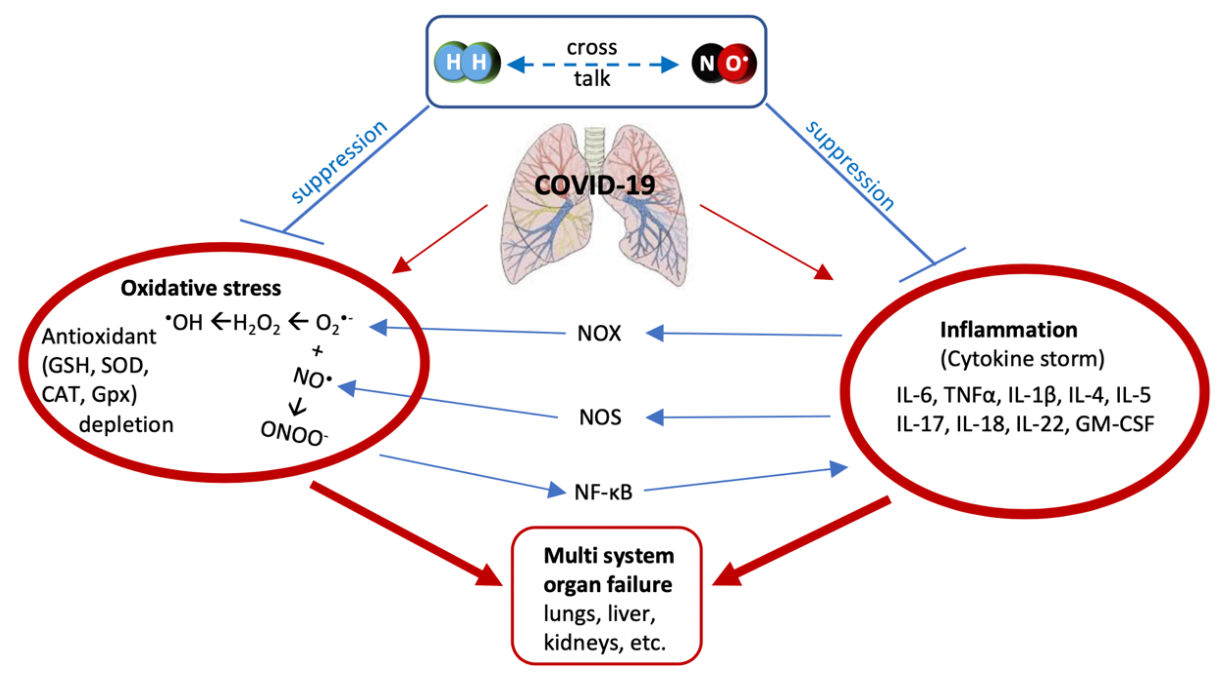

\section{Introduction}

Coronavirus disease-19 (COVID-19) is a highly infectious disease caused by the severe acute respiratory syndrome coronavirus 2 (SARS-CoV-2), which resulted in the current 2019-20 coronavirus pandemic [1]. Although the virus can harm many organs, the lungs are the primary ones most affected. In addition to flu-like symptoms such as fever, fatigue, and shortness of breath, COVID-19 may also
${ }^{*}$ Correspondence to: Tyler W. LeBaron, Centre of Experimental Medicine, Institute for Heart Research, Slovak Academy of Sciences, Bratislava, Slovak Republic and Molecular Hydrogen Institute, USA, E-mail: lebaront@ molecularhydrogeninstitute.com

Received: March 19, 2020; Accepted: March 27, 2020; Published: April 01, 2020 
progress to pneumonia, multi-organ failure, and even death [1]. These sequelae are consequences of pathogen-induced cellular aberrations that further arise from and result in oxidative and inflammatory stress responses. Even if the immune system can overcome the infection, the concomitant multi-organ damage may result in additional distress and increased mortality. Accordingly, favorable regulation of the immune system and the associated inflammatory and oxidative response is optimal. Currently, there is no vaccine or other clearly effective medical intervention to treat COVID-19 [1]. However, along with many interventions and drugs being tested, at least four clinical trials are investigating the use of molecular hydrogen in patients suffering with COVID-19, and are reporting positive preliminary results (RefA).

HydroShot is a novel, zero-caloric, functional beverage containing nitric-oxide-stimulating citrulline that has been infused with molecular hydrogen. The concentration of $\mathrm{H}_{2}$ is above $2 \mathrm{ppm}$ as determined via gas chromatography $\left(\mathrm{H}_{2}\right.$ Analytics, Las Vegas, USA; SRI 8610C; California USA). All ingredients have a high safety profile and have GRAS (Generally Recognized as Safe) designation by the USFDA. Although HydroShot is primarily considered a "healthy beverage" and is regularly consumed by a healthy demographic, HydroShot may serve as an effective modality to improve human physiology including the cardiovascular and immune systems. Correspondingly, it may play an important role in the global battle against both communicable and non-communicable diseases including COVID-19. We briefly review some of the literature regarding molecular hydrogen and nitric oxide/ citrulline as it relates to this global COVID-19 pandemic. Lastly, we provide some preliminary data for improved blood flow following HydroShot consumption, several favorable case reports, and finally suggest that in addition to is frequent consumer use, clinical research on HydroShot should be conducted for COVID-19 and other diseases.

\section{Molecular hydrogen and immune function}

Molecular hydrogen $\left(\mathrm{H}_{2}\right)$, is an odorless, highly diffusible, flammable gas, and is naturally produced by intestinal bacteria [2]. A 1975 publication in Science by researchers at Texas A\&M and Baylor University reported that hyperbaric hydrogen was an effective treatment for squamous cell carcinoma in albino mice [3]. Later, a 2007-Nature Medicine publication demonstrated that inhalation of $\mathrm{H}_{2}$ markedly suppressed the brain damage in a rat stroke model by suppressing oxidative stress and inflammation [4]. Subsequent scientific publications $(\approx 1500)$ and human clinical trials $(\approx 80)$ from universities and institutions around the world have further corroborated the initial finding that $\mathrm{H}_{2}$ has therapeutic effects [5]. Molecular hydrogen is administered to humans primarily either via inhalation or dissolved in water to create hydrogen-rich water (HRW) [5]. In Japan, molecular hydrogen has been approved as an advanced medicine for the treatment of post-cardiac arrest syndrome, for which a 360 patient, multi-center, clinical study is being conducted [6]. $\mathrm{H}_{2}$ has anti-oxidant, anti-inflammatory, anti-allergy, and anti-apoptotic protective effects. Although the exact molecular mechanism(s) remain elusive, $\mathrm{H}_{2}$ affords these protective effects by modulating signal transduction, influencing gene expression, and modulating protein-phosphorylation cascades [5]. These favorable effects of molecular hydrogen may uniquely qualify it as an optimal therapy against COVID-19 and similar diseases. Indeed, $\mathrm{H}_{2}$ has already been proposed to be an effective treatment for multiple organ dysfunction induced by influenza and other viral infectious diseases based on the current animal and human publications [7].

For example, in zebrafish challenged with the bacterium Aeromonas hydrophila, HRW down-regulated pro-inflammatory genes (IL-1 $\beta$, IL-
6 , and NF- $\mathrm{B}$ ) and up-regulated the anti-inflammatory cytokine IL10. Furthermore, HRW increased the survival of the zebrafish, and effectively blocked replication of the bacterium [8]. Similarly, in a rat model of lipopolysaccharide (LPS)-induced systemic inflammation, HRW significantly reduced plasma pro-inflammatory mediators (i.e. TNF- $\alpha$, IL- 6 prostaglandin $\mathrm{E}_{2}$ ), and increased the anti-inflammatory cytokine, IL-10. Symptomatically, $\mathrm{H}_{2}$ prevented LPS-induced hypotension, fever [9], neuroinflammation, and promoted recovery from LPS-induced sickness [10].

$\mathrm{H}_{2}$ therapy effectively suppressed radiation-induced damage to the immune system such as preventing the decline in the number of splenocytes and the number of $\mathrm{CD}^{+}$and $\mathrm{CD} 8^{+} \mathrm{T}$ lymphocytes. Moreover, hydrogen prevented the radiation-induced imbalance between Th1, T2, Th17 and Treg cells, as well as other cytokines (TNF- $\alpha$, transforming growth factor- $\beta 1$, GM-CSF, IL- $1 \beta$, IL-4, IL-5, IL-10, IL-17, IL-23) [11]. Similarly, in a human study of 55 stage IV colorectal cancer patients, molecular hydrogen inhalation restored exhausted $\mathrm{CD}^{+} \mathrm{T}$ cells [12]. These cytotoxic T lymphocytes target cells that are infected with cancer and viruses [13]. Another randomized human clinical study in patients with chronic hepatitis $B(n=60)$ found that ingestion of HRW for 6 weeks reduced oxidative stress, and tended to suppress the viral DNA level and improved liver function [14].

It has also been demonstrated that oral HRW abolishes the immediate-type allergic reaction in mice, and prevents degranulation of mast cells by suppressing NADPH oxidase activity [15]. The researchers subsequently reported that HRW reduced LPS/interferon gammainduced NO production in macrophages, which form a molecular basis for the functional interaction between these two gaseoussignaling molecules [16]. The mechanisms responsible for these antiinflammatory and anti-oxidant effects appear to at least partly involve hydrogen's ability to significantly suppress the induction of the NLRP3 inflammasome [17], and activate the Nrf2-antioxidant pathway [5], and cytoprotective heme oxygenase-1 [18]. In addition to the high cellular bioavailability of the $\mathrm{H}_{2}$ molecule, its immunomodulating activity coupled with its ability to restore homeostasis to the dysregulation of cytokines (i.e. prevention/attenuation of cytokine storm), gives it a significant therapeutic advantage over other medical interventions [5].

\section{Nitric oxide and immune function}

Like molecular hydrogen, nitric oxide $\left(\mathrm{NO}^{*}\right)$ is also a gaseoussignaling molecule. Nitric oxide is long recognized as one of the most versatile and key regulators of the immune system. It is involved in the pathogenesis and control of autoimmune processes and infectious diseases [19]. Moreover, it has been demonstrated to efficiently inhibit the replication cycle of the severe acute respiratory syndrome coronavirus in vitro [20]. Congruously, nitric oxide is being clinically evaluated in COVID-19 patients (RefB).

Nitric oxide is primarily produced via nitric oxide synthase (NOS), of which there are three isoforms, namely, inducible NOS (iNOS), neuronal NOS (nNOS), and endothelial NOS (eNOS) [21]. The amino acid arginine is metabolized by this enzyme to produce $\mathrm{NO}^{*}$ and citrulline. NO${ }^{*}$ levels gradually decrease with age; up to $75 \%$ in 70-80-year-olds compared to healthy 20 -year-olds [22]. Excessive inflammatory conditions result in multiple organ dysfunction due to misdistribution of blood flow and perturbations of microcirculation [21]. This occurs essentially due to a NO deficiency in needed locations with an excessive production in non-needed locations. Arginine availability is often significantly impaired in inflammatory conditions such as in septic patients [21]. Arginine deficiency may 
occur due to its increased catabolism and limited citrulline availability, which is used for arginine de novo synthesis. Since even under normal conditions, arginine is quickly metabolized, research demonstrates that supplementation with citrulline is more effective at increasing plasma arginine and $\mathrm{NO}$ levels compared to supplementing with arginine [23]. Low levels of citrulline are associated with acute respiratory distress syndrome, and may also lead to NOS uncoupling, which induces further oxidative and cellular damage [24].

\section{Molecular hydrogen and nitric oxide combination}

Nitric oxide, as a reactive free radical, encourages either cell survival or cell death depending on its concentration, location, and timing of production. Importantly, molecular hydrogen regulates nitric oxide ( $\mathrm{NO}^{*}$ ) levels via both reducing excessive production (e.g. suppressing iNOS activity) and also increasing production (e.g. enhancing eNOS activity) [5]. Therefore, NO regulation is critical to potentiate its beneficial therapeutic effects and mitigate its harmful effects. NO reacts nearly instantaneously with superoxide $\left(\mathrm{O}_{2}^{*}\right)$ to form pernicious peroxynitrite ( $\left.\mathrm{ONOO}^{-}\right)$, which is an extremely oxidative and cytotoxic molecule [25]. The reaction with $\mathrm{O}_{2}{ }^{-}$- directly lowers the availability of circulating $\mathrm{NO}$, which further exacerbates the diseased condition [25]. The radicals, $\mathrm{O}_{2}{ }^{-}$and $\mathrm{NO}{ }^{*}$, have important beneficial roles in the immune system including killing pathogens, inhibiting their replication, and regulating the inflammatory response. However, under stressful conditions, such as with COVID-19 and its progression to pneumonia, multi-organ failure, and other sequelae, these molecules lose their critical regulation and contribute to the etiology and pathology of these diseases forming both ONOO- and the extremely toxic hydroxyl radical $(\cdot \mathrm{OH})$ [25]. Molecular hydrogen has been demonstrated to favorably regulate the production of both $\mathrm{O}_{2}{ }^{*}$ and $\mathrm{NO} \cdot$ by influencing NADPH oxidase, and the NOS enzymes (iNOS, eNOS and nNOS) [5]. Additionally, $\mathrm{H}_{2}$ can effectively reduce the toxic hydroxyl and peroxynitrite oxidants [4]. Furthermore, combination therapy of $\mathrm{NO}^{*}$ and $\mathrm{H}_{2}$ demonstrated a synergistic effect in an acute lung-injury model such as what can occur with COVID-19. The treatment significantly attenuated lung neutrophil recruitment, inflammation, and apoptosis induced by LPS and polymicrobial sepsis [26]. Moreover, $\mathrm{H}_{2}$ prevented $\mathrm{NO}^{-}$-induced damage as evidenced by the elimination of nitrotyrosine levels seen with NO therapy alone [26]. This adds credence to the concept that oral ingestion of HydroShot, a hydrogen-infused functional beverage containing NO'-producing citrulline, may provide significant benefits to the immune system.

\section{HydroShot preliminary data}

Increased nitric oxide production: Due to the nitric-oxide stimulating capability of HydroShot, its ingestion accordingly increases endogenous production of nitric oxide as seen in Figure 2. The nitric oxide levels were increased from being depleted at baseline to the optimal level in $30 \mathrm{~min}$, followed by a slight gradual trend upwards at hours 1, 3, 5 and 8 from baseline testing (Figure 2a). Since the test strips are not quantitative and have upper and lower limits, the absolute changes, peak, and duration are unknown. However, it is clear that the nitric oxide levels were increased to optimal levels for at least eight hours following HydroShot ingestion.

Similarly, using the FDA approved NIOX VERO machine (Aerocrine AB, Solna, Sweden) the increased nitric oxide levels are also observed (Figure 2b). NIOX VERO is a medical device that measures fractional exhaled nitric oxide (FeNO*), which is a commonly used to help diagnose and manage asthma in children and adults [27]. The baseline FeNO' of 12 subjects (six men and six women; age $45.3 \pm$
21.1) were obtained, and then they ingested $\mathrm{H}_{2} \mathrm{Bev} \mathrm{H}_{2}$-infused $\mathrm{NO}$ stimulating product and their FeNO' levels were measured again 45 min later. The product resulted an average of $202.49 \%$ increase in FeNO levels. Interestingly, when HRW was ingested alone, the $\mathrm{NO} \cdot$ levels slightly decreased. This may be due to hydrogen's ability to influence the regulation of $\mathrm{NO}^{*}$ production and decrease its overexpression. Asthmatic patients present with abnormally elevated $\mathrm{NO} \cdot$ levels due to an overactive immune response. Molecular hydrogen has been demonstrated to decrease airway inflammation in allergic asthmatic mice [28]. Anecdotally, asthmatic consumers have reported beneficial relief from ingestion of molecular hydrogen, but cite greater therapeutic effects from ingestion of the $\mathrm{NO}^{-}$-stimulating product. For example, a 12-year-old female reported an average Peak Flow Meter Reading at baseline of $220 \mathrm{~L} / \mathrm{min}$. However, following $15 \mathrm{~min}$ of product ingestion, the flow meter increased by $15 \%$. Finally, after three months of daily use, the baseline flow meter increased to $350 \mathrm{~mL} / \mathrm{min}$ ( $160 \%$ increase).

Blood flow: As mentioned earlier, nitric oxide also promotes blood flow by inducing vasodilation. Optimal blood perfusion is critical for normal organ function, wound healing, and exercise performance.

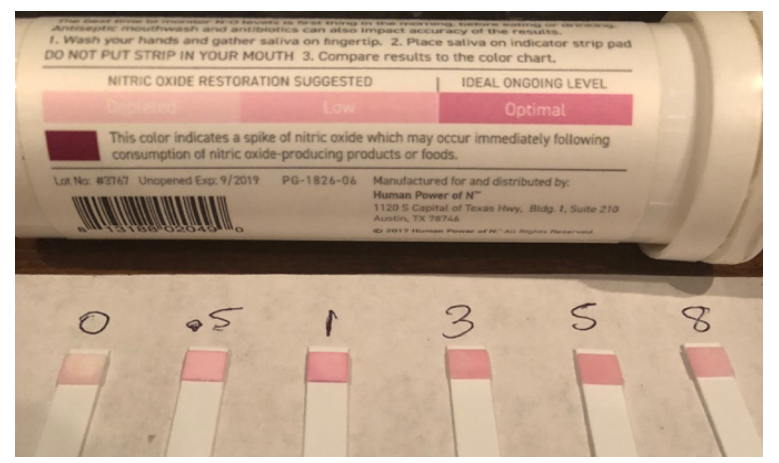

(a)

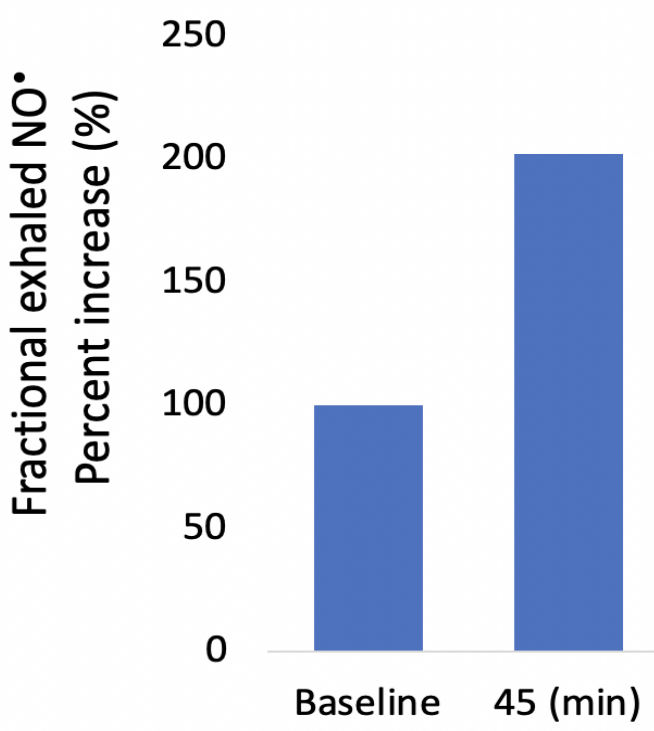

(b)

Figure 2. (a) Increased nitric oxide production following ingestion of product. Baseline (far left) depleted nitric oxide levels; $0.5,1,3,5$, and $8 \mathrm{hrs}$ after ingestion optimal nitric oxide levels. (b) Increased nitric oxide production following ingestion of product. (b) represents the average percent increase of $\mathrm{FeNO} \bullet$ by 12 subjects following product ingestion after 45 min compared to baseline 
Blood flow provides oxygen, nutrients, hormones, signaling metabolites, etc. to the cells, and is necessary to remove harmful metabolic waste products. Similar to nitric oxide levels, blood flow also decreases with age [29], which may contribute to neurodegeneration, neuropathy [30], and an impaired immune system [31]. In line with the previous data on increased nitric oxide production, Figure 3 illustrates the average preliminary data (triplicate measurements) of blood flow following the ingestion of HydroShot. The blood flow significantly increased from baseline at 15 minutes and remained elevated above baseline for over 15 hrs compared to control. The peak increase (231\%) occurred approximately five hrs after ingestion.

The increased blood flow was also reflected in thermal imaging of the hand as shown in Figure 4. Baseline hand temperature averaged 96.8 degrees, and after 30 minutes it increased to $99.2 \mathrm{~F}$.

The increased blood flow is likely due to a combination of molecular hydrogen and the other ingredients (e.g. citrulline) in the HydroShot. The increase in blood flow and the reflective thermal imaging was only mildly noticeable and for a shorter duration when either hydrogen alone or citrulline alone was ingested (data not shown). This may suggest that there may be a synergistic effect between these two clinically relevant molecules.

Wound healing: In line with the increased blood flow, there have been several reports of increased healing following ingestion of HydroShot. Figure 5 depicts a 72 -year old male with skin cancer who underwent Mohs surgery followed by 28 radiation treatments. He drank two cans of HydroShot daily while undergoing Mohs surgery, radiation, and recovery. As illustrated in the pictures, the skin healed at an accelerated rate. The radiation-induced skin discoloration was forecasted to heal in approximately two to three months, but instead healed in only 10 days.

Figure 6 shows ankle pictures of a male who required a skin graft following a surgical operation. Over 6 months, the first two skin grafts failed, and the third also started to fail. It was recommended to perform amputation the following week. However, the patient began daily ingestion of HydroShot, and there was noticeable improvement the following day. Shortly after, the skin graft took and was successful. The tunneling hole completely healed after 7 weeks of daily HydroShot ingestion.

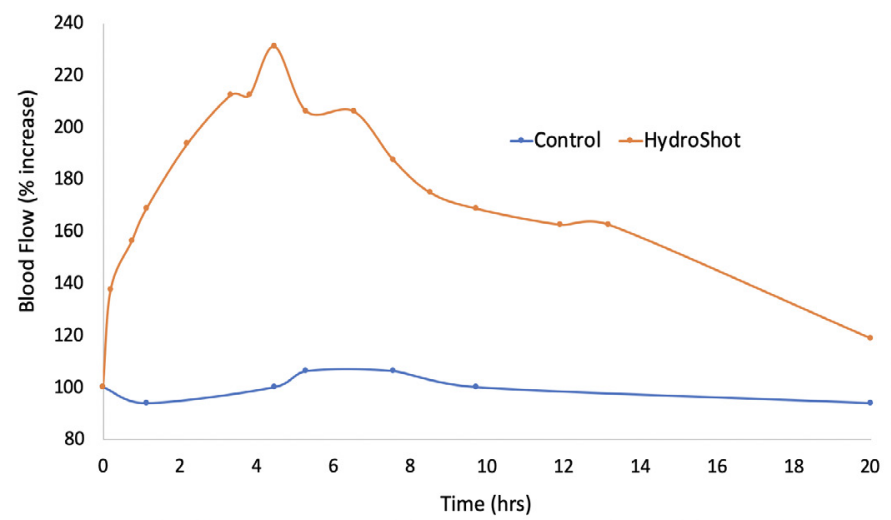

Figure 3. Changes in blood flow over 20 hours following ingestion of HydroShot compared to its starting baseline and control. HydroShot increased blood flow by $231 \%$ at five hours followed by a gradual decline towards baseline. Control values for blood flow did not significantly change compared to its starting baseline

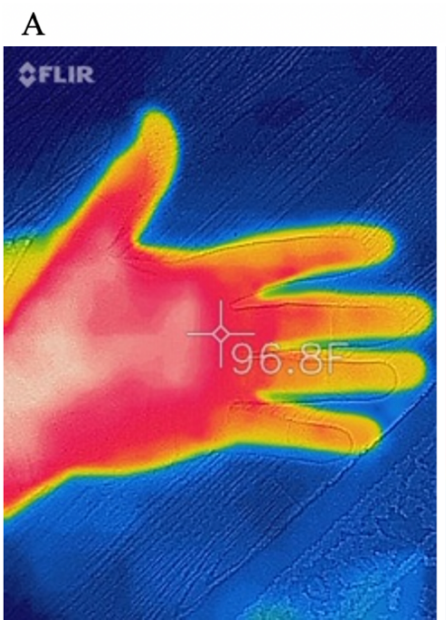

B

Figure 4. Thermal imaging of hand temperature following ingestion of HydroShot Baseline temperature was $96.8 \mathrm{~F}(\mathrm{~A}) ; 30 \mathrm{~min}$ later temperature increased to $99.2 \mathrm{~F}(\mathrm{~B})$.

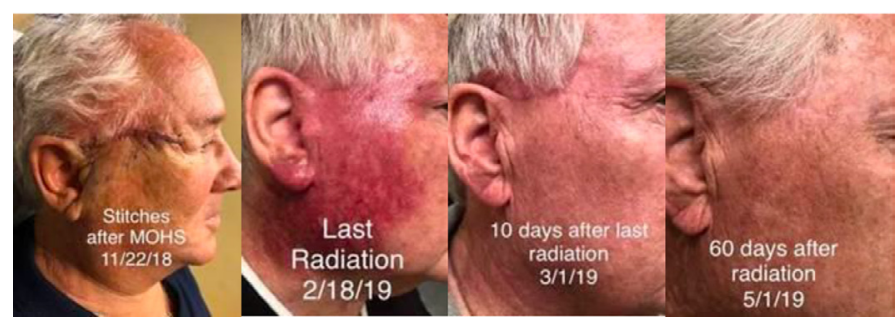

Figure 5. Effects of daily HydroShot ingestion during Mohs surgery, radiation treatment and recovery. HydroShot accelerated healing in 10 days compared to the predicted two to three months
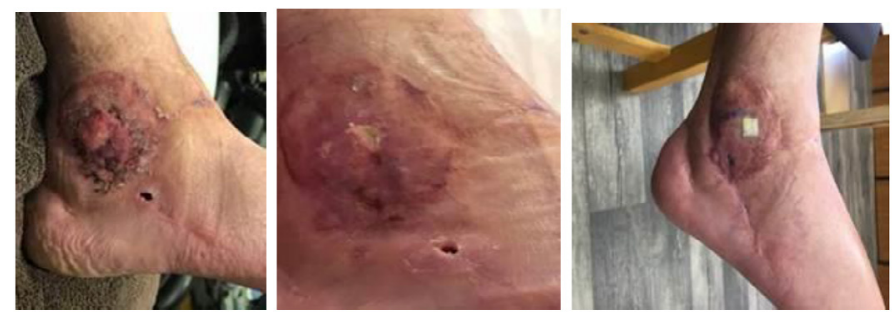

Figure 6. Effects of HydroShot ingestion following three attempts of unsuccessful skin grafts. After ingestion of HydroShot, the skin graft was successful, and the wound was healed

\section{Discussion and conclusion}

The biomedical effects of molecular hydrogen and nitric oxide are well-substantiated in the scientific literature. Indeed, these molecules have been demonstrated in pre-clinical and clinical studies to exert a wide range of therapeutic effects. In line with our data, previous research has demonstrated that high-concentration HRW could improve endothelial function and significantly increase flow-mediated dilation of the brachial artery [32]. Similarly, the increased healing effects of HydroShot are corroborated by previous research where administration of HRW via tube-feeding to severely hospitalized elderly patients with pressure ulcers resulted in wound size reduction, and a faster recovery [33]. However, when compared to the preliminary data from HydroShot, these results suggest that the addition of citrulline significantly increases the therapeutic action (i.e. blood flow and wound healing) of the beverage. 
At the cellular level, COVID-19 and similar diseases are characterized by dysregulated inflammatory response of the immune system, excessive oxidative stress, and impaired cellular function. Nitric oxide plays a dual role in actively improving the immune system, destroying pathogens, inhibiting their replication, and also in promoting cellular oxidative damage and promotion of excessive inflammation. However, since nitric oxide plays a key role in healthy cellular function, inhibiting or activating the NOS enzymes via pharmacological intervention similarly has both beneficial and harmful consequences [25]. In contrast, molecular hydrogen is a novel and safe medical gas that affords therapeutic effects by regulating redox status and the inflammatory response [5]. In addition to the clinically relevant antioxidant and anti-inflammatory effects of $\mathrm{H}_{2}$, it also has the ability to regulate nitric oxide production and attenuate its adverse effects [5].

Currently, inhalation of $\mathrm{H}_{2}$ gas is being used in at least four clinical studies [RefA]. Dr. Zhong Nanshan, the epidemiologist who discovered the SARS coronavirus in 2003, recently recommended the inhalation of molecular hydrogen for COVID-19 patients based on the preliminary clinical data [RefC]. Hydrogen treatment resulted in significant amelioration of dyspnea in most patients with COVID-19 in the pilot investigation. Correspondingly, hydrogen inhalation is included as a recommendation by the National Health Commission of The People's Republic of China's protocol for COVID-19 [RefD]. Although their research is on inhalation of molecular hydrogen, ingestion of HRW is also effective, and, at least in some cases, more effective [34]. Moreover, the addition of citrulline to boost nitric oxide levels, both of which can be in deficiency during late stages of COVID-19, further give credence to its observed therapeutic effects in the presented preliminary data. In conclusion, due to the urgency of the COVID-19 pandemic, and the simplicity, safety, and clinical viability of HydroShot, it is recommended that HydroShot, in addition to its frequent consumer use as a healthy beverage, be clinically investigated for its potential use in treating COVID-19 patients.

\section{Author contributions}

TWL helped analyze the data, and organized and prepared the manuscript; MLM provided medical advice, technical insights, and assisted with the data testing; KHR developed the hydrogen products, participated in data acquisition, and provided insights for manuscript. All authors read and approved the manuscript.

\section{References}

1. Hui DS (2020) The continuing 2019-nCoV epidemic threat of novel coronaviruses to global health - The latest 2019 novel coronavirus outbreak in Wuhan, China. Int J Infect Dis 91: 264-266.

2. Suzuki Y (2009) Are the effects of alpha-glucosidase inhibitors on cardiovascular events related to elevated levels of hydrogen gas in the gastrointestinal tract? FEBS Letters 583: 2157-2159.

3. Dole M, Wilson FR, Fife WP (1975) Hyperbaric hydrogen therapy: a possible treatment for cancer. Science 190: 152-154.

4. Ohsawa I (2007) Hydrogen acts as a therapeutic antioxidant by selectively reducing cytotoxic oxygen radicals. Nat Med 13: 688-694.

5. LeBaron TW (2019) A New Approach for the Prevention and Treatment of Cardiovascular Disorders. Molecular Hydrogen Significantly Reduces the Effects of Oxidative Stress. Molecules 24.

6. Tamura T (2017) Efficacy of inhaled HYdrogen on neurological outcome following BRain Ischemia During post-cardiac arrest care (HYBRID II trial): study protocol for a randomized controlled trial. Trials 18: 488.

7. Yang M (2017) Hydrogen Medicine Therapy: An Effective and Promising Novel Treatment for Multiple Organ Dysfunction Syndrome (MODS) Induced by Influenza and Other Viral Infections Diseases? SOJ Microbiology \& Infectious Diseases 5: 1-6.
8. Hu Z (2017) Impact of molecular hydrogen treatments on the innate immune activity and survival of zebrafish (Danio rerio) challenged with Aeromonas hydrophila. Fish Shellfish Immunol 67: 554-560.

9. Saramago EA (2019) Molecular hydrogen potentiates hypothermia and prevents hypotension and fever in LPS-induced systemic inflammation. Brain Behav Immun 75 119-128.

10. Spulber S (2012) Molecular hydrogen reduces LPS-induced neuroinflammation and promotes recovery from sickness behaviour in mice. PLoS One 7: e42078.

11. Zhao S (2014) Protective effect of hydrogen-rich saline against radiation-induced immune dysfunction. J Cell Mol Med 18: 938-946.

12. Akagi J and Baba H (2019) Hydrogen gas restores exhausted CD8+ T cells in patients with advanced colorectal cancer to improve prognosis. Oncol Rep 41: 301-311.

13. Appay V, Douek DC, Price DA (2008) CD8+ T cell efficacy in vaccination and disease. Nat Med 14: 623-628.

14. Xia C (2013) Effect of hydrogen-rich water on oxidative stress, liver function, and viral load in patients with chronic hepatitis B. Clin Transl Sci 6: 372-375.

15. Itoh T (2009) Molecular hydrogen suppresses FcepsilonRI-mediated signal transduction and prevents degranulation of mast cells. Biochem Biophys Res Commun 389: 651-656.

16. Itoh T (2011) Molecular hydrogen inhibits lipopolysaccharide/interferon gammainduced nitric oxide production through modulation of signal transduction in macrophages. Biochemical and Biophysical Research Communications 411: 143-149.

17. Ren JD (2016) Molecular hydrogen inhibits lipopolysaccharide-triggered NLRP3 inflammasome activation in macrophages by targeting the mitochondrial reactive oxygen species. Biochim Biophys Acta 1863: 50-55.

18. Chen HG (2013) Heme oxygenase-1 mediates the anti-inflammatory effect of molecular hydrogen in LPS-stimulated RAW 264.7 macrophages. Int J Surg 11: 1060-1066.

19. Bogdan C (2001) Nitric oxide and the immune response. Nat Immunol 2: 907-916.

20. Akerstrom S (2005) Nitric oxide inhibits the replication cycle of severe acute respiratory syndrome coronavirus. J Virol 79: 1966-1969.

21. Wijnands KA (2015) Arginine and citrulline and the immune response in sepsis. Nutrients 27: 1426-1463.

22. Torregrossa AC, Aranke M, Bryan NS (2011) Nitric oxide and geriatrics: Implications in diagnostics and treatment of the elderly. J Geriatr Cardiol 8: 230-242.

23. Schwedhelm E (2008) Pharmacokinetic and pharmacodynamic properties of oral L-citrulline and L-arginine: impact on nitric oxide metabolism. Br J Clin Pharmacol 65: 51-59.

24. Ware LB (2013) Low plasma citrulline levels are associated with acute respiratory distress syndrome in patients with severe sepsis. Crit Care 17: R10.

25. Pacher P, Beckman JS, Liaudet L (2007) Nitric oxide and peroxynitrite in health and disease. Physiol Rev 87: 315-424.

26. Liu H (2015) Combination therapy with nitric oxide and molecular hydrogen in a murine model of acute lung injury. Shock 43: 504-511.

27. Arnold RJ (2018) A Review of the Utility and Cost Effectiveness of Monitoring Fractional Exhaled Nitric Oxide (FeNO) in Asthma Management. Manag Care 27: $34-41$.

28. Zhang N (2018) Inhalation of hydrogen gas attenuates airway inflammation and oxidative stress in allergic asthmatic mice. Asthma Res Pract 4: 3 .

29. Khan AS (2001) Growth hormone increases regional coronary blood flow and capillary density in aged rats. J Gerontol A Biol Sci Med Sci 56: B364-B371.

30. Sonntag WE (1997) Decreases in cerebral microvasculature with age are associated with the decline in growth hormone and insulin-like growth factor 1. Endocrinology 138: 3515-3520.

31. Khaddaj Mallat R (2017) The vascular endothelium: A regulator of arterial tone and interface for the immune system. Crit Rev Clin Lab Sci 54: 458-470.

32. Sakai T (2014) Consumption of water containing over $3.5 \mathrm{mg}$ of dissolved hydrogen could improve vascular endothelial function. Vasc Health Risk Manag 10: 591-597.

33. Li Q (2013) Hydrogen water intake via tube-feeding for patients with pressure ulcer and its reconstructive effects on normal human skin cells in vitro. Med Gas Res 3: 20.

34. Ito M (2012) Drinking hydrogen water and intermittent hydrogen gas exposure, but not lactulose or continuous hydrogen gas exposure, prevent 6-hydorxydopamine-induced Parkinson's disease in rats. Med Gas Res 2: 15. 
LeBaron TW (2020) A novel functional beverage for COVID-19 and other conditions: Hypothesis and preliminary data, increased blood flow, and wound healing

35. RefA: https://www.cebm.net/oxford-covid-19_trashed/covid-19-registered-trialsand-analysis/?fbclid=IwAR2TnDJspDYcrF-yeq3cv2wtLTfd7vbgIo4VIT340tjz9AN5BiRJJnp-z0

36. RefB: NCT04290871: Nitric Oxide Gas Inhalation for SARS in COVID-19. (NOSARSCOVID)
37. RefC: Perspectives of the management of COVID-19 infection in China (EWS webinar serious)

38. RefD: New Coronavirus Pneumonia Diagnosis and Treatment Scheme (Trial Ver7: Mar $3,2020)$

Copyright: (C2020 LeBaron TW. This is an open-access article distributed under the terms of the Creative Commons Attribution License, which permits unrestricted use, distribution, and reproduction in any medium, provided the original author and source are credited. 